TITLE:

\title{
Piezoresistive properties of carbon nanotubes under radial force investigated by atomic force microscopy
}

\section{$\operatorname{AUTHOR}(\mathrm{S})$ :}

Nishio, Taichi; Miyato, Yuji; Kobayashi, Kei; Matsushige, Kazumi; Yamada, Hirofumi

\section{CITATION:}

Nishio, Taichi ... [et al]. Piezoresistive properties of carbon nanotubes under radial force investigated by atomic force microscopy. APPLIED PHYSICS LETTERS 2008, 92(6): 063117.

\section{ISSUE DATE:}

2008-02-11

URL:

http://hdl.handle.net/2433/84579

\section{RIGHT:}

Copyright 2008 American Institute of Physics. This article may be downloaded for personal use only. Any other use requires prior permission of the author and the American Institute of Physics. 


\title{
Piezoresistive properties of carbon nanotubes under radial force investigated by atomic force microscopy
}

\author{
Taichi Nishio, ${ }^{1}$ Yuji Miyato, ${ }^{1}$ Kei Kobayashi, ${ }^{2, a)}$ Kazumi Matsushige, ${ }^{1}$ and \\ Hirofumi Yamada ${ }^{1,3}$ \\ ${ }^{1}$ Department of Electronic Science and Engineering, Kyoto University, Katsura, Nishikyo, Kyoto 615-8510, \\ Japan \\ ${ }_{2}^{2}$ Innovative Collaboration Center, Kyoto University, Katsura, Nishikyo, Kyoto 615-8520, Japan \\ ${ }^{3}$ Core Research for Evolutional Science and Technology, Japan Science and Technology Agency, Katsura, \\ Nishikyo, Kyoto 615-8510, Japan
}

(Received 27 October 2007; accepted 19 January 2008; published online 14 February 2008)

\begin{abstract}
We investigated the piezoresistive properties of single-wall carbon nanotubes (SWCNTs) under the tip-induced force in the radial direction using atomic force microscopy. We found that the conductance of the bundled SWCNTs was modulated by the applied radial force. The polarity and amount of the conductance change were different on every bundle and even dependent on the location where the force was applied. These phenomena were explained by the modulation of the band structures of the SWCNTs, which was caused by the deformation at the critical pressure.

(C) 2008 American Institute of Physics. [DOI: 10.1063/1.2857480]
\end{abstract}

The carbon nanotube (CNT), especially the single-wall carbon nanotube (SWCNT), is one of the most promising building blocks for nanometer-scale electronic devices. CNTs have been shown to have metallic or semiconducting electrical transport characteristics depending on their chiralities, ${ }^{1}$ as was predicted by theoretical studies. Metallic CNTs are one of the candidate materials for via interconnects in the integrated circuits, ${ }^{2}$ utilizing their high current capacity and high thermal conductivity. There have also been many studies on the carbon nanotube field-effect transistors (CN-FETs) using semiconducting CNTs as a channel. ${ }^{3-7}$

It is already known that the electrical transport in CNTs is strongly affected by the geometrical perturbations such as defects. ${ }^{8}$ There is an increasing number of research studies on the effect of the external forces on the transport properties of CNTs for applications in nanometer-scale electromechanical systems. There were some pioneering measurements of the electrical transport properties under mechanical stress. ${ }^{9-15}$ The effects of the axial, ${ }^{9-11}$ torsional, ${ }^{12}$ radial $^{13,14}$ and flexural ${ }^{15}$ strains were investigated and the conductance changes were measured. In most of these studies, the CNTs were suspended and their transport properties were measured while the forces were applied using insulating atomic force microscopy (AFM) tips. ${ }^{9,10}$ This method, however, has a drawback such that the effects of the axial strain and the radial strain cannot be separated. In order to overcome this problem, Gómez-Navarro et al. employed a different approach in that they measured the current of the CNTs lying on a hard insulating substrate. ${ }^{13,14}$ They used a metallized tip as a tool to apply radial forces and on the electrodes to measure the transport characteristics. This makes it difficult to separate the effect of the radial deformation and that from the change in the contact resistance. We now introduce an alternative method to measure the piezoresistive properties of the CNTs by applying an external force in the radial direction using an insulating AFM tip.

${ }^{a)}$ Electronic mail: keicoba@iic.kyoto-u.ac.jp.
We prepared CN-FET structures using the dielectrophoresis technique. ${ }^{16}$ A pair of electrodes $(12 \mathrm{~nm} \mathrm{Au} / 3 \mathrm{~nm} \mathrm{Cr})$ with $2 \mu \mathrm{m}$ gap was prepared on a highly doped $\mathrm{Si}$ substrate with a 300-nm-thick oxide. The SWCNTs, purchased from Bucky USA (arc-discharge method), were dispersed in ethanol and then dropped onto the gap, while an ac electric field $\left(1 \mathrm{MHz}, 10 \mathrm{~V}_{p-p}\right)$ was applied. After drying, we found electrode pairs which only have a single bridged bundle of SWCNTs by AFM. The transport characteristics were measured in vacuum $\left(10^{-2} \mathrm{~Pa}\right)$.

We used an AFM apparatus (JEOL: JSPM-5200) and a lightly doped $\mathrm{Si}$ cantilever (Olympus OMCL-AC160TS), whose nominal spring constant was $42 \mathrm{~N} / \mathrm{m}$. Since it was not easy to precisely locate the position of the tip on the SWCNTs due to the thermal drift of the sample translation stage, we measured the current versus applied force characteristics at each point in an area including the SWCNTs and their surrounding surface. The measurement was realized by modifying the measurement procedure for the current imaging tunneling spectroscopy. Figure 1 shows a schematic diagram of the measurement procedure. The distance between the tip and sample was regulated by the intermittent-contact mode in which the vibration amplitude was kept constant. We obtained a topographic image consisting of 128 $\times 128$ pixels by a raster scan. At each pixel, the tip-sample

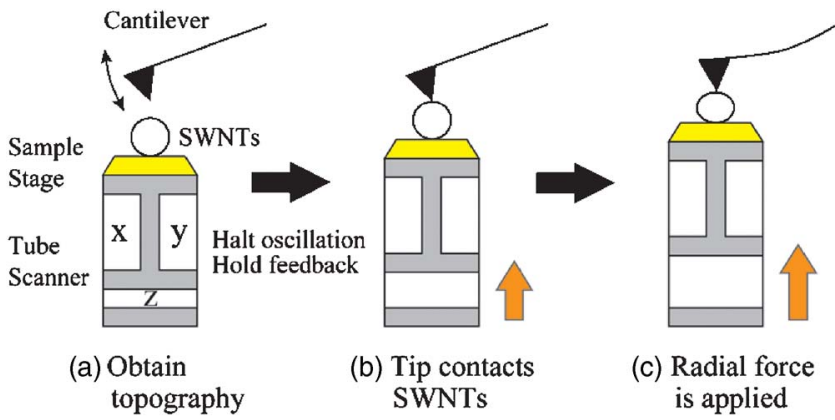

FIG. 1. (Color online) Schematic diagram of procedure for measurement of piezoresistive properties of SWCNTs by applying a radial force using AFM cantilever tip. 

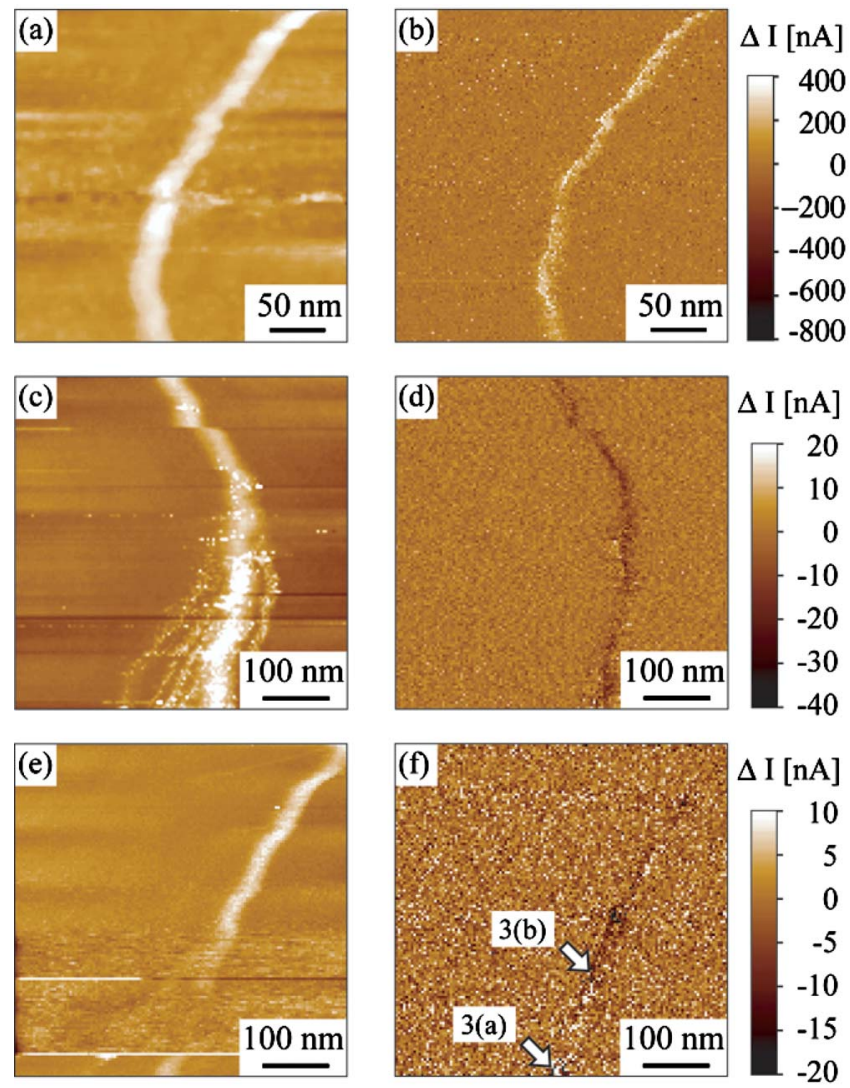

FIG. 2. (Color online) Three sets of topographic images and the differential current image measured at a specific load. (a), (c), and (e) are the topographic images and (b), (d), and (f) are the differential current images, which were simultaneously taken on the same area, respectively.

distance was held for a certain period by the sample-hold circuit [Fig. 1(a)]. Upon holding the tip-sample distance, the excitation signal for the tip vibration was also halted. The tip vibration gradually decreased to zero. The sample stage was translated toward the tip by applying a ramp voltage to the piezoelectric tube scanner [Figs. 1(b) and 1(c)]. During translating the sample stage, a bias voltage was applied between the $\mathrm{Au}$ electrodes, and the current flowing through the SWCNTs was measured by an electrometer (Keithley: 6487). After the measurement of the current-to-load characteristic, the sample stage was translated back to its prior position, and the tip vibration and the tip-sample distance was resumed. From the collected current-to-load characteristics at each pixel, we can obtain the current image at each load. All measurements were performed in a 1 atm nitrogen atmosphere.

Figures 2(a) and 2(b) are the topographic image and its corresponding differential current image at the load of $350 \mathrm{nN}$. The diameter of the bundled SWCNTs was about $3.2 \mathrm{~nm}$. The bias voltage was $0.5 \mathrm{~V}$ and the conductance without the loading force $\left(G_{0}\right)$ was about $220 \mathrm{nS}$. The latter image was obtained by subtracting the current image at the zero loading force from that with the load. Figures 2 (c) and 2(d) are the set of images taken on a different bundle. The diameter of the bundle was about $4.8 \mathrm{~nm}$. The bias voltage was $50 \mathrm{mV}$ and $G_{0}$ was about $11 \mu \mathrm{S}$. The differential current image [Fig. 2(d)] shows the conductance change for the load of $1 \mu \mathrm{N}$.

These results indicate that the conductance of the bundled SWCNTs was modulated by the applied radial force. However, the polarity and amount of the conductance change Downloaded 29 Jun 2009 to 130.54.110.22. Redistribution subject
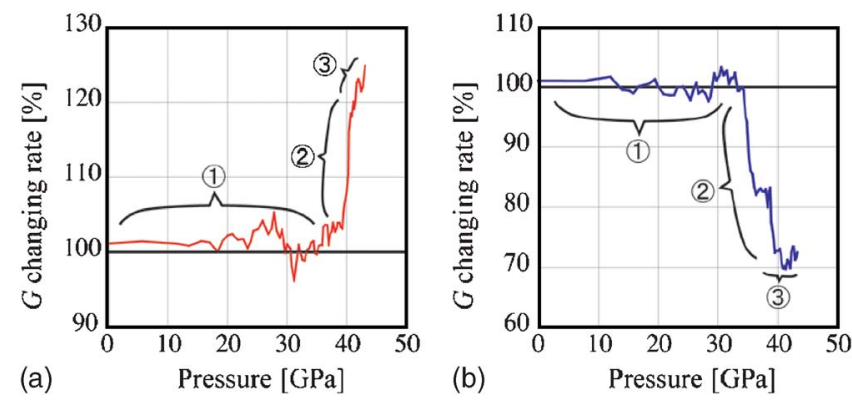

FIG. 3. (Color online) Plots of the conductance change normalized to that measured without the load $\left(G_{0}\right)$ as a function of the applied pressure, which was calculated using the Hertz model, for the case when the conductance was increased (a) and decreased (b) upon increasing the pressure.

were different for every bundle, as shown in Figs. 2(b) and 2(d). The SWCNTs in Fig. 2(b) exhibited an increase in the conductance, and the SWCNTs in Fig. 2(d) showed a decrease. We also found that some SWCNTs showed both bright and dark contrasts in the differential current image, as shown in Figs. 2(e) and 2(f). The diameter of the bundle was about $3.7 \mathrm{~nm}$. The bias voltage was $50 \mathrm{mV}$ and $G_{0}$ was about $5 \mu \mathrm{S}$. The differential current image [Fig. 2(f)] shows the conductance change for the load of $1 \mu \mathrm{N}$.

As a further discussion, the applied force $(F)$ was converted to a pressure $(P)$ using the following equation, the Hertz model for a cylinder in contact with a sphere, ${ }^{17}$ $P=(C / \pi)\left(6 F E^{* 2} / R^{2}\right)^{1 / 3}$, where $E^{*}$ and $R$ are effective Young's modulus and the equivalent radius of curvature, respectively. $C$ is a correction factor for cylinder and sphere, which is determined by their radii. $E^{*}$ and $R$ were defined by the following equations: $E^{*}=\left[\left(1-v_{\text {tip }}^{2}\right) / E_{\text {tip }}+(1\right.$ $\left.\left.-v_{\mathrm{CNT}}^{2}\right) / E_{\mathrm{CNT}}\right]^{-1}$ and $R=\left(R_{\text {tip }}^{-1}+R_{\mathrm{CNT}}^{-1}\right)^{-1}$. Young's modulus and Poisson's ratio for the tip are $E_{\text {tip }}=166 \mathrm{GPa}$ and $v_{\text {tip }}=0.217,{ }^{18}$ and those for the CNTs are $E_{\mathrm{CNT}}=30 \mathrm{GPa}$ (Ref. $19)$ and $v_{\mathrm{CNT}}=0.27,{ }^{20}$ respectively. The radius of the CNTs, $R_{\mathrm{CNT}}$, is assumed as half the measured height in the AFM topography, which is actually the radius of the bundle. We assume the tip radius, $R_{\text {tip }}$, as $30 \mathrm{~nm}$ from the measured line profile of the bundle in the AFM topography. The correction factor $C$ for these radii is about 1.2. ${ }^{17}$

Figure 3 shows two plots of the differential conductance normalized to $G_{0}$ depending on the applied loading pressure, measured on the point indicated by the arrows in Figs. 2(e) and 2(f). As illustrated in Fig. 3, the measured curve can be divided into three regimes showing (1) almost no change, (2) a sharp increase or decrease, and (3) a gradual change.

According to the molecular dynamics simulations, ${ }^{21-23}$ both the bundled SWCNTs and isolated SWCNT show abrupt structural changes around a certain pressure, which is called the critical pressure $\left(P_{c}\right)$. The SWCNT is expected to deform into an elliptical shape from its original circular shape around the $P_{c}$. The value of $P_{c}$ depends on the diameter of the SWCNT, but it is on the order of several GPa. The existence of $P_{c}$ was proved by Raman spectroscopy ${ }^{24}$ and $\mathrm{X}$-ray diffraction measurements. ${ }^{25}$

As shown in Fig. 3, we observed an abrupt conductance change at the pressure of about 35 or $40 \mathrm{GPa}$. We speculated that the observed conductance change is related to the deformation of the SWCNTs. Therefore, the observed three regimes correspond to (1) below the $P_{c},(2)$ around the $P_{c}$, and (3) above the $P_{c}$. The difference in the value of $P_{c}$ from the o AlP license or copyright; see http://apl.aip.org/apl/copyright.jsp 
theoretical studies can be attributed to the fact that these simulations are for the SWCNTs under a uniform pressure, such as the hydrostatic pressure and the pinching between two parallel plates, while the tip-induced radial force is not always uniform. Application of the simple Hertz model for converting the applied force to the pressure may be another source of error.

In our experiments, we measured the sum of the conductance change in all the SWCNTs in the bundle. The conductance change in the individual SWCNTs can be explained by one of the following mechanisms. It was predicted by Lammert et al. that the metallic CNTs become semiconducting as a result of the coupling between the opposing internal faces. ${ }^{26}$ It was also reported by Kiliç et al. that semiconducting nanotubes become metallic under radial deformation due to the enhanced $\pi^{*}-\pi^{*}$ hybridization. ${ }^{27}$ Capaz et al. calculated $d E_{g} / d P$ ( $E_{g}$ is the band gap width and $P$ is the pressure) for several SWCNTs with the so-called zigzag chiralities, and showed that the band gap will be modulated by the pressure. ${ }^{28}$ We consider that the bundle contained both metallic and semiconducting SWCNTs. Therefore, some bundles showed an increase while the others showed a decrease. It is even possible that the bundle shows either an increase or decrease depending on the location at which the force is applied if the bundle is not uniform. We actually found that some SWCNTs have their ends in the halfway of the bundle. It was not possible to determine the detailed structures of the bundle in our experiments. It would be desirable to bridge the single SWCNT whose chirality is determined by some other techniques (scanning tunneling microscopy, for example) and to measure its piezoresistive property, which will be our future task.

In summary, we investigated the piezoresistive properties of the bundled SWCNTs under a tip-induced radial force. Our results showed both a conductance increase and decrease depending on the samples and on the position at which the radial force was applied. The conductance change went through three regimes. These three regimes can be explained by the existence of the critical pressure $P_{c}$ at which the CNT deforms. The deformation causes a modulation of the band structure, which leads to the conductance change.

This work was supported by a Grant-in-Aid for Scientific Research and Grants for Regional Science and Technology Promotion from the Ministry of Education, Culture, Sports, Science and Technology of Japan, and by the Core Research for Evolutional Science and Technology (CREST) of the Japan Science and Technology Agency (JST).
${ }^{1}$ C. Dekker, Phys. Today 52, 22 (1999).

${ }^{2}$ F. Kreupl, A. P. Graham, G. S. Duesberg, W. Steinhogl, M. Liebau, E. Unger, and W. Honlein, Microelectron. Eng. 64, 399 (2002).

${ }^{3}$ S. J. Tans, A. R. M. Verschueren, and C. Dekker, Nature (London) 393, 49 (1998).

${ }^{4}$ F. Nihey, H. Hongo, Y. Ochiai, M. Yudasaka, and S. Iijima, Jpn. J. Appl. Phys., Part 2 42, L1288 (2003).

${ }^{5}$ A. Javey, H. Kim, M. Brink, Q. Wang, A. Ural, J. Guo, P. Mcintyre, P. McEuen, M. Lundstorm, and H. Dai, Nat. Mater. 1, 241 (2002).

${ }^{6}$ Y. Li, S. Bangsaruntip, X. Wang, L. Zhang, Y. Nishi, and H. Dai, J. Am. Chem. Soc. 128, 3518 (2006).

${ }^{7}$ B. M. Kim, T. Brintlinger, E. Cobas, M. S. Fuhrer, H. Zheng, Z. Yu, R. Droopad, J. Ramdani, and K. Eisenbeiser, Appl. Phys. Lett. 84, 1946 (2004).

${ }^{8}$ V. H. Crespi, M. L. Cohen, and A. Rubio, Phys. Rev. Lett. 79, 2093 (1997).

${ }^{9}$ T. W. Tombler, C. Zhou, L. Alexseyev, J. Kong, H. Dai, L. Liu, C. S. Jayanthi, M. Tang, and S. Y. Wu, Nature (London) 405, 769 (2000).

${ }^{10}$ E. D. Minot, Y. Yaish, V. Sazonova, J.-Y. Park, M. Brink, and P. L. McEuen, Phys. Rev. Lett. 90, 156401 (2003).

${ }^{11}$ J. Cao, Q. Wang, and H. Dai, Phys. Rev. Lett. 90, 157601 (2003).

${ }^{12}$ T. Cohen-Karni, L. Segev, O. Srur-Lavi, S. R. Cohen, and E. Joselevich, Nat. Nanotechnol. 1, 36 (2006).

${ }^{13}$ C. Gomez-Navarro, P. J. D. Pablo, and J. Gomez-Herrero, Adv. Mater. (Weinheim, Ger.) 16, 549 (2004).

${ }^{14}$ C. Gomez-Navarro, J. J. Sáenz, and J. Gomez-Herrero, Phys. Rev. Lett. 96, 076803 (2006).

${ }^{15}$ V. Semet, V. T. Binh, D. Guillot, K. B. K. Teo, M. Chhowalla, G. A. J. Amaratunga, W. I. Milne, P. Legagneux, and D. Pribat, Appl. Phys. Lett. 87, 223103 (2005).

${ }^{16}$ Y. Miyato, K. Kobayashi, K. Matsushige, and H. Yamada, Jpn. J. Appl. Phys., Part 1 44, 1633 (2005).

${ }^{17}$ K. L. Johnson, Contact Mechanics (Cambridge University Press, Cambridge, 1985).

${ }^{18}$ K. Yamanaka and S. Nakano, Appl. Phys. A: Mater. Sci. Process. 66, S313 (1998).

${ }^{19}$ I. Palaci, S. Fedrigo, H. Brune, C. Klinke, M. Chen, and E. Riedo, Phys. Rev. Lett. 94, 175502 (2005).

${ }^{20}$ J. P. Lu, Phys. Rev. Lett. 79, 1297 (1997).

${ }^{21}$ J. A. Elliott, J. K. W. Sandler, A. H. Windle, R. J. Young, and M. S. P. Shaffer, Phys. Rev. Lett. 92, 095501 (2004).

${ }^{22}$ X. H. Zhang, D. Y. Sun, Z. F. Liu, and X. G. Gong, Phys. Rev. B 70, 035422 (2004).

${ }^{23}$ D. Y. Sun, D. J. Shu, M. Ji, F. Liu, M. Wang, and X. G. Gong, Phys. Rev. B 70, 165417 (2004).

${ }^{24}$ U. D. Venkateswaran, A. M. Rao, E. Richter, M. Menon, A. Rinzlere, R. E. Smalley, and P. C. Eklund, Phys. Rev. B 59, 10928 (1999).

${ }^{25}$ S. M. Sharma, S. Karmaker, S. K. Sikka, P. V. Teredesai, A. K. Sood, A. Govindaraj, and C. N. R. Rao, Phys. Rev. B 63, 205417 (2001).

${ }^{26}$ P. E. Lammert, P. Zhang, and V. H. Crespi, Phys. Rev. Lett. 84, 2453 (2000).

${ }^{27}$ Ç. Kılıç, S. Ciraci, O. Gülseren, and T. Yildirim, Phys. Rev. B 62, R16345 (2000).

${ }^{28}$ R. B. Capaz, C. D. Spataru, P. Tangney, M. L. Cohen, and S. G. Louie, Phys. Status Solidi B 241, 3352 (2004). 